\title{
Localisation and Mapping Using a Laser Range Finder: A Goal-Seeking Approach
}

\author{
Sotirios Ch. Diamantas and Richard M. Crowder \\ School of Electronics and Computer Science \\ University of Southampton \\ Southampton, SO17 1BJ, United Kingdom \\ $\{$ sd04r, rmc $\} @$ ecs.soton.ac.uk
}

\begin{abstract}
In this paper we examine the problem of localisation and mapping of an unknown environment using data from a laser range finder. In order to support our method we detect landmarks in the environment using the same laser finder. For the localisation and mapping process to take place we assume that the mobile robot will follow a path until a landmark is observed by the laser scanner. Our approach alleviates the requirement to provide odometry or other information. In addition, an efficient path is sought to reach target location. An inherent property of this is obstacle avoidance. The simulated experiments presented in the paper validate the effectiveness of our approach.
\end{abstract}

\section{Introduction}

For a mobile robot to build a map of an unknown environment an accurate estimation of the position of the robot is required as well as a means for effectively mapping the environment. Localisation and mapping can be considered to be a "a chicken and egg" problem, requiring both accurate position estimates of the robot and of the surroundings. A laser range finder is highly suitable for that purpose due to its strength in estimating distances accurately while unburdening the system from problems arising in other sensors like infrared or sonar. In fact, there is a large number of works that utilise laser range sensors for obstacle avoidance $[19,2,12,14]$.

In this work no prior information about the environment is assumed. The only hypothesis made is that the robot knows where it should head to. Thus, we feedback the robot with a goal that has to be sought. However, even this hypothesis can be ruled out so long as the robot navigates without any restrictions, i.e., without having to pursue an effective path. In such a case the robot can navigate towards places where there are distinguishable points by the laser finder, for example corners, and drive among such 'places'.

In this paper we present a navigation method based on the idea of Vector Field Histogram (VFH) [19]. The robot scans the environment using the laser sensor and based on the measurements taken an efficient path is sought. In order to infer an efficient path the target point is assumed to be known. We can, therefore, make a supposition that the goal position is known by its $x, y, \phi$ coordinates or that the goal position can be seen through a vision sensor and an estimate of its distance or direction can be taken. Next, the robot will try to identify 'distinctive' landmarks in the scanned environment; these are mainly corners, and can be identified by the range of the neighbouring ray values. The robot will thus extract the shortest obstacle-free path to the target defined by the rays of laser scanner. While traversing the chosen route, the landmark is tracked down by the laser scanner's rays until it is 'met' by the side rays of it. We use the side rays of the laser scanner to identify the landmark as they form a $90^{\circ}$ angle with the chosen path. The sides of the right triangle formed by the side rays of the scanner, the landmark, and the position of the robot at which the landmark was first detected are computed with the trigonometric functions.

The process of finding a 'distinctive landmark' is critical for the path to be extracted as is needed to be as close to the autonomous agent as possible. Fig. 1 demonstrates this process where the robot at time $t_{0}$ scans the environment and selects the nearest landmark to it, depicted by the bold scan line. At time $t_{1}$ the robot is scanning again the environment using the laser scanner and an updated second path is chosen to reach the target at point denoted by the ' $\mathrm{X}$ ' symbol. The robot could have missed to reach the target and end up in a blind alley had it not selected a nearby landmark.

The primary purpose of this work is to tackle one of the core problems in robotics science, namely localisation and mapping using minimal sensing. It is not uncommon that 
a critical sensor to the system may fail and thus having to bypass it using alternative sensors and methods. Such cases become of high importance if the autonomous agent acts in remote or hostile environments. Moreover, sensors like GPS cannot operate in environments like, indoors, underwater, or the outer space. Therefore, we have approached the localisation and mapping problem using only a laser scanner sensor to infer accurate maps of the environment while at the same time localising the robot.

This paper is comprised of five sections. Following is section 2 where related work is presented. Then, in section 3 we present and analyse the methods that have been implemented to localise the robot and map the unknown environment. In section 4 we present and discuss the experimental results. Finally, section 5 epitomizes the paper with a brief discussion on the conclusions derived from this work and a future work.

\section{Related work}

Localisation and mapping has been at the forefront of robotics research the last decade. The work presented in this paper is closely related to the Simultaneous Localisation and Mapping (SLAM) [16] problem in which a robot has to build map while at the same time estimating its position relative to the map. Most of the approaches make use of Kalman Filter (KF) and Extended Kalman Filter (EKF) $[6,13],[3,4]$. Under these methods a matrix representing the robot and landmarks' position is established. As the number of landmarks increases so does the matrix resulting in a computational expensive solution. An alternative solution to KF and EKF is the use of particle filters [8] and Monte Carlo methods [17, 18].

FastSLAM $[11,15]$ is another method that integrates particle filters and Extended Kalman Filters. FastSLAM tries to alleviate from the problem of data association, that is, the problem where landmarks look alike which is prevalent in the previous methods. Most of the above mentioned methods use mainly odometry information and laser sensors to support the SLAM methodology. Nonetheless, there has also been a SLAM-based method that employs a single camera [5] to infer the 3D trajectory of a monocular camera in an unknown environment. This vision-based SLAM is widely known for years as the 'structure from motion' problem and it has been researched in parallel and in ignorance of the robotics community. Other sensors which have been used for localisation include Radio Frequency Identification (RFID) technology. For example [10], proposes a model based on RFID technology and a laser-based FastSLAM approach to effectively determine the location of RFID tags.

In [1] the authors proposed a method for map building without using any odometry information. Their method

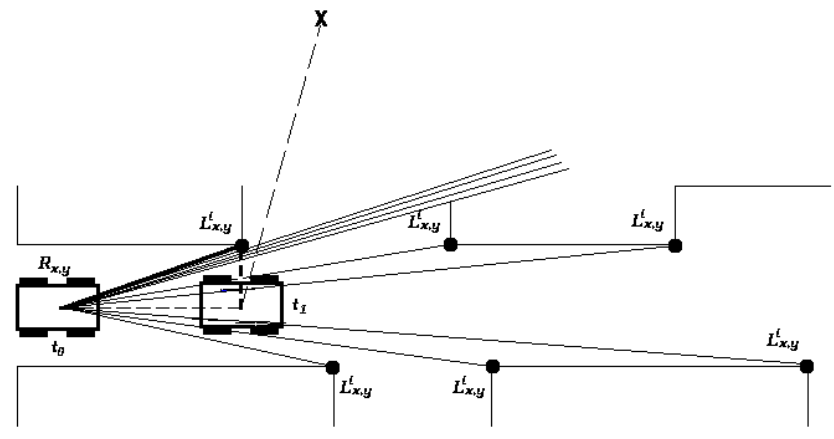

Figure 1. Robotic agent having selected the nearest landmark which leads to an efficient path to the target point ' $X$ '.

builds a geometrical global map based on various scans of the environment taken at different instants of time. They try to integrate the maps using three different methods, namely sequential, tree, and pivot methods. However, they do not keep track of the position of the manually driven robot. An approach which uses Dynamic Programming (DP) for the real-time self-localisation of a robot is explained in [7]. In this work a panorama laser range finder (PLRF) is employed. Similarly to the previous work, a matching between present preprocessed scans and already stored scan data is taking place. The task of preprocessing method is to extract line segments from the acquired range data. Moreover, in their approach they make use of local coordinate systems linked together by topological information. No global environmental map is built, but they rather focus on local 'distinctive' places to build local maps for self-localisation.

\section{Methodology}

In this section we describe the methods we have followed to tackle the problem of localisation and mapping. No apriori knowledge of the environment is provided. However, as already stated we adopt a goal-seeking approach and a competent path is selected which is defined by the laser scanner rays. The map of the environment is initially empty and is built up as the navigation of the autonomous agent proceeds. Fig. 2 depicts the structure of the localisation and mapping algorithm.

During the first phase the robot collects raw data from the laser range finder, and in the next one the obstacles of the environment are expanded, see Fig. 3, according to the radius, $r$, of the robot using the equation of the circle (1), 


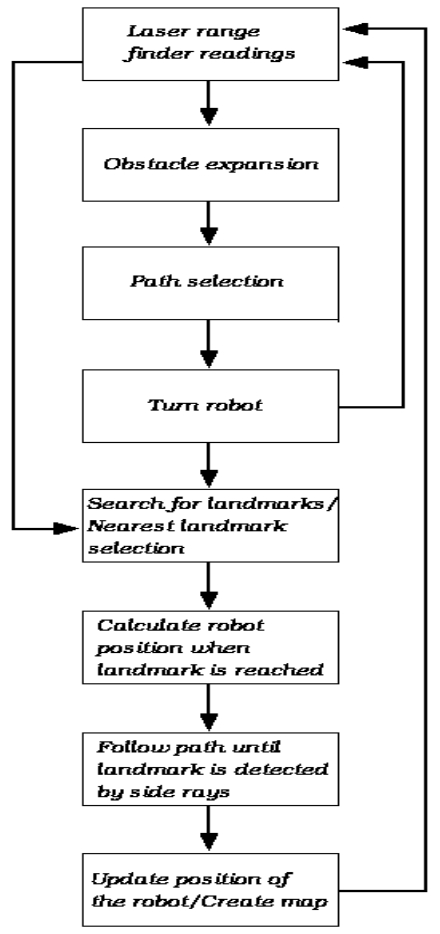

Figure 2. Flowchart of the various stages of the localisation and mapping algorithm.

$$
(x-h)^{2}+(y-k)^{2}=r^{2},
$$

where $h, k$ is the centre of the circle $C$, in this case the point at which the ray of the laser hits on the obstacle, and the equation of the line (2),

$$
y=m x+b,
$$

that represents a laser ray. We compute the slope (3) of the line, i.e., ray,

$$
m=\frac{C_{k}-R_{y}}{C_{h}-R_{x}}
$$

by knowing the current position of the robot $R_{x, y}$ and the end position of the ray, i.e., $C_{h, k}$. Next, we substitute equation (2) into the second part of (1) having (4),

$$
(x-h)^{2}+(m x+b-k)^{2}=r^{2} .
$$

Thus, from (4) we end up having a quadratic equation (5),

$$
a x^{2}+b x+c=0,
$$

which we solve it in order to obtain the points in which the rays intersect (or hit) the obstacles expanded by the radius $r$. Thus, if discriminant, $\Delta>0$, then the ray intersects the

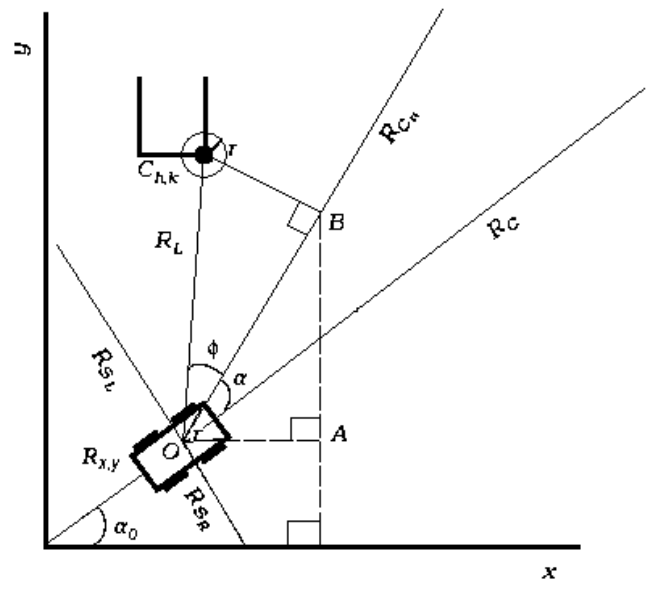

Figure 3. Robotic agent having identified a new path, $R_{C_{n}}$, and a landmark, $C_{h, k}$. This figure shows how the localisation coordinates are computed from the triangles $O B C$ and $O A B$.

expanded obstacle in two points, whereas if $\Delta=0$, there exists a tangent ray to the circle. If $\Delta<0$ then the ray is not intersecting any point of the circle formed by the expansion of the obstacle. Fig. 4 shows the rays of the laser scanner one of which hits at an obstacle with coordinates $x=2$, $y=2$. The circle represents the expansion of the obstacle by the radius, $r$, of the robot, in this case 0.3 metres. The marked lines representing the laser rays that fall within the expanded obstacle do not thus provide a safe path for the robot. All other rays, hence paths, would provide a safe path if they were to be taken. Fig. 5 shows the outcome of applying the above algorithm to the laser data with added noise.

Upon expansion of obstacles the path selection module takes over, Fig. 2, and an efficient path is chosen based on the proximity of the laser ray to the target point. As written earlier, the coordinates of the target are known to the system. This however, could have been omitted had we used a compass or a vision sensor to know at any instant of time the direction to the target. So long as the path is selected, the robot rotates around its axis until its central ray, $R_{C}$, Fig. 3, points to the selected path, $R_{C_{n}}$. The next step involves updating the laser data by taking a new laser scan after the robot has rotated by angle $\alpha$. At this point the landmark search module is taking over which identifies corners, jumps, and discontinuities in the laser scan. Such points act as 'landmarks' and are identified when a ray being equal to the maximum range, say set $A$, and its neigh- 


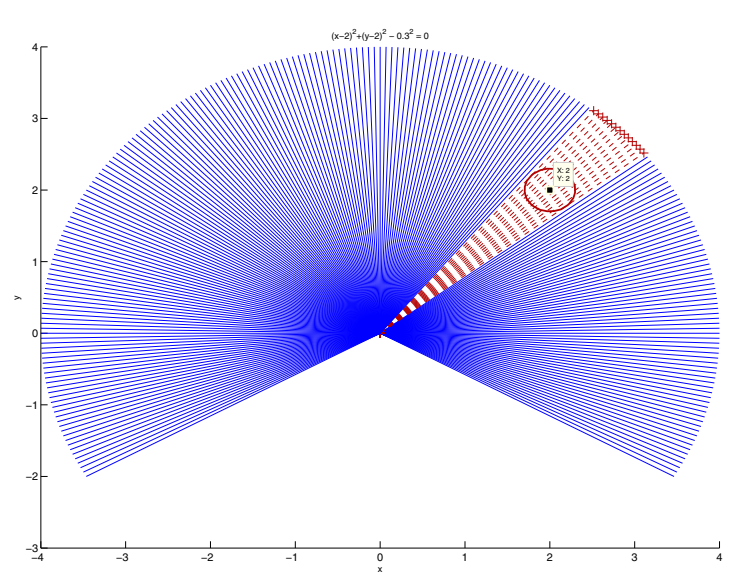

Figure 4. A simulated scan sample with a detectable area of $240^{\circ}$ and a detectable distance of 4.0 metres. An expanded point by 0.3 metres is shown with the laser rays falling into the circle being marked.

bouring $n$ rays do not belong to the set $A$, i.e., they hit on an obstacle. Therefore, the mathematical formulation of this is, $\operatorname{ray}_{x} \in A$, and $\operatorname{ray}_{x+n} \notin A$ or $\operatorname{ray}_{x-n} \notin A$ constitute a 'landmark'.

Furthermore, the landmark search module will eventually select a single landmark at each time step for navigation. The selection of this landmark is based upon its proximity to robot location. This is happening because a shorter path to the target may be discovered after the approximation of the agent to the landmark. Therefore, we use the Euclidean distance, $d$, between the landmark location, $\left(L_{x}^{i}\right.$, $\left.L_{y}^{i}\right)$, and the robot, $\left(R_{x}, R_{y}\right)$ to find the nearest landmark. Fig. 1 shows an example of landmark selection among a set of detected landmarks. The Euclidean distance is described by (6)

$$
d=\min i \sqrt{\left(R_{x}-L_{x}^{i}\right)^{2}+\left(R_{y}-L_{y}^{i}\right)^{2}} .
$$

The area of searching for landmarks is less than $90^{\circ}$ from the central ray, $R_{C}$, Fig. 3, as the robot is using its side rays, namely, $R_{S_{L}}$ and $R_{S_{R}}$, to detect the landmark and build a map. At this point we use a local frame of reference to calculate distances to landmarks. Fig. 3 shows how the method works. The robot after having inferred a new path it rotates by angle $\alpha$, that is, from $R_{C}$ to $R_{C_{n}}$. At this point its $R_{L}$ ray detects and selects landmark $C_{h, k}$. The sides of the triangle $O B C$ named $C B$ and $O B$ are calculated using the trigonometric functions $\sin \phi$ and $\cos \phi$.

Having found the coordinates of local frame of reference

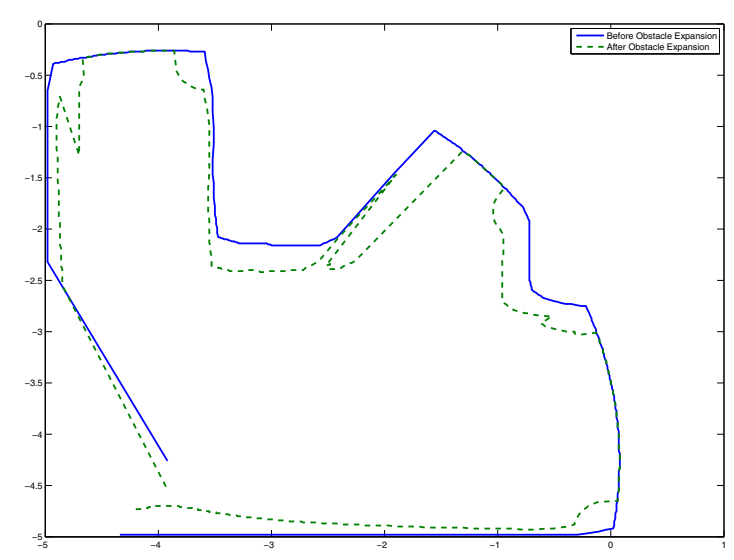

Figure 5. Expansion of obstacles.

we add them up to the global frame of reference. Again, Fig. 3 depicts the variables involved. This is done by adding the angle by which the agent has rotated, that is $\alpha$ degrees, to initial pose $\alpha_{0}$, thus inferring the pose of the robot. We then find the projection of side $O B$ to the $x$-axis and $y$-axis. The sides of the triangle $O A B$ are again calculated with the cosine and sine trigonometric functions. Their outcome will thus give us the $x, y$ coordinates of the point $B$ which are added to the initial $R_{x, y}$ coordinates of the robot.

In order for the robot to follow the path from point $O$ to point $B$ the landmark at point $C_{h, k}$ is tracked down by the neighbouring rays of the $R_{L}$ ray as the robot proceeds. At point $B$, as Fig. 3 shows, the side ray, $R_{S_{L}}$, will detect the landmark by the range of the $R_{S_{L}}$ ray which should approximately be equal to $C B$ found earlier. Due to noise, it is quite probable that the landmark be missed. For this reason we have simulated a laser scanner whose field-ofview (FOV) is $240^{\circ}$ so if the landmark is missed by the side ray, $R_{S_{L}}$, it can be detected by the neighbouring rays with angle larger than $90^{\circ}$. Fig. 6 shows this scenario.

At this time step the robot will localise itself with its global $x, y$ coordinates and create a new map of the environment. Having reached the final step of the algorithm, the agent initiates a new laser scan to proceed with the navigation process.

\section{Experimental results}

The localisation and mapping algorithm has been developed on the Player/Stage 2D simulation environment [9]. The simulated devices consist of an Erratic mobile robot and a HOKUYO URG04LX laser range finder with $240^{\circ}$ field of view, 4.0 metres maximum range, and 685 samples 


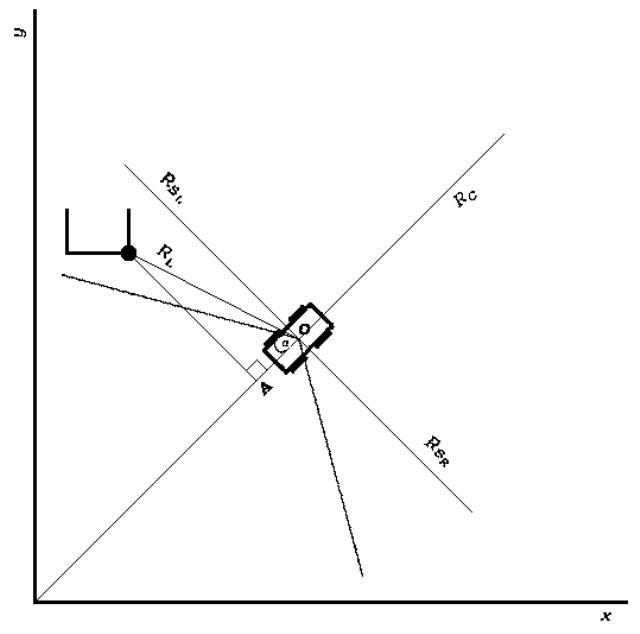

Figure 6. An example of how the location of the robot can be calculated after having missed the landmark by the side ray, $R_{S_{L}}$. The landmark is, however, still detectable by the neighbouring rays of the laser scanner.

per scan. The angular resolution was set to $0.35^{\circ}$. Fig. 7 shows a snapshot of the simulated testing environment, the size of which is 10.0 metres by 10.0 metres. The robot is on the left lower corner with a delineation of the laser scanner.

Fig. 8 shows the first scan at position $R_{x_{0}}=-3.92$, $R_{y_{0}}=-4.26$. The target position has been set at $x=4.00$, $y=4.00$. In this first laser scan there have been detected six landmarks of which, the nearest one, is selected for reference which is at position $x=-2.38, y=-2.06$. The robot will try to re-detect the landmark from position denoted by the symbol $\times$ at location $x=-2.09, y=-2.34$.

In Fig. 9 the robot has successfully recognised the landmark and has updated its location coordinates; a new scan has performed with two landmarks present one of which again is selected for the navigation.

In Fig. 10 the robot has traversed the environment even more and has detected two other landmarks. At this point, although the target position is at $x=4.00, y=4.00$, the robot has selected its next targeted position, denoted by $\times$ symbol, to be further upwards instead of being on its right side. This occurs because the expansion of the obstacles obscures some laser rays that form a path in the right-hand side of the robot. The arrow shows the position, in particular

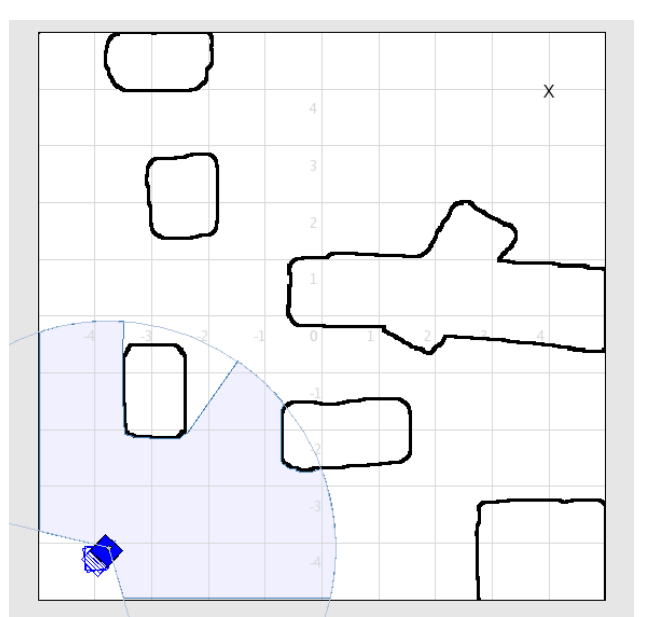

Figure 7. Snapshot of the simulated environment.

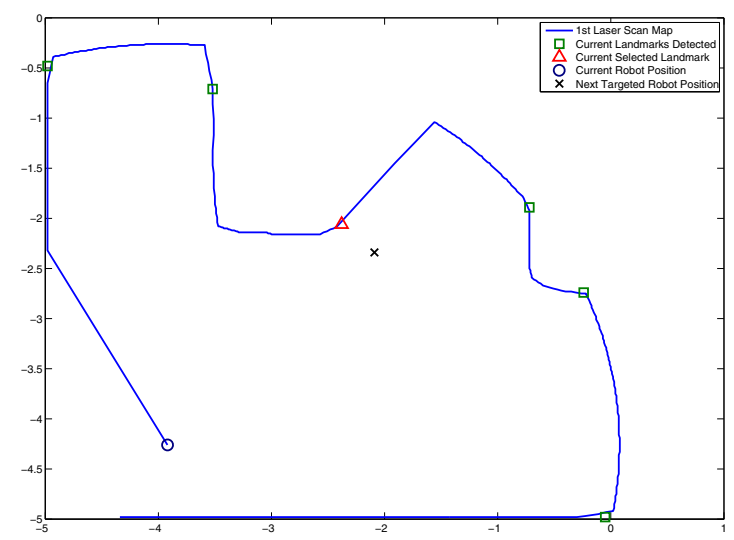

Figure 8. First scan of the environment.

the corner point, at which this occurs.

It should be noted that during the landmark selection process we use the map that does not contain the expanded obstacles. The map with the expanded obstacles is used in the path selection process. In Fig. 11 the robot has reached its desired position and a new scan has been taken which has detected two landmarks in the environment, one of which is again selected.

In Fig. 12 the robotic agent has almost reached its target. The boldface $\times$ denotes the target position. However, in this figure we see the influence of noise into the navigation process. There exists a map drift between the previous laser scan and the current one. This noise effect has caused the agent to select its next target point a little further away than 


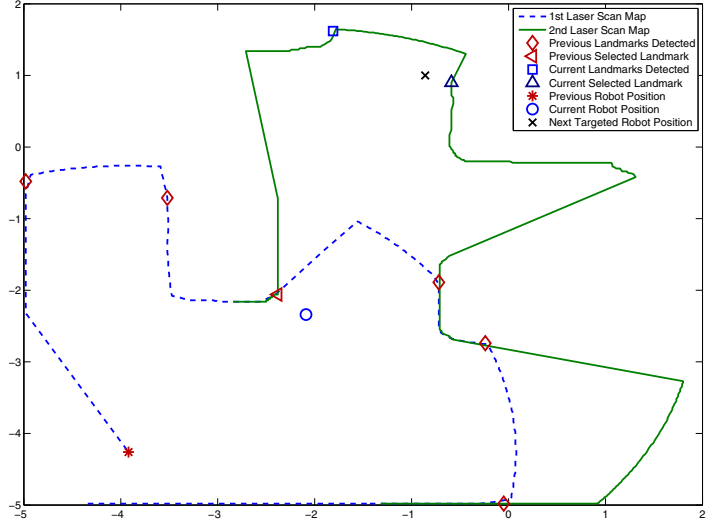

Figure 9. Second scan of the environment.

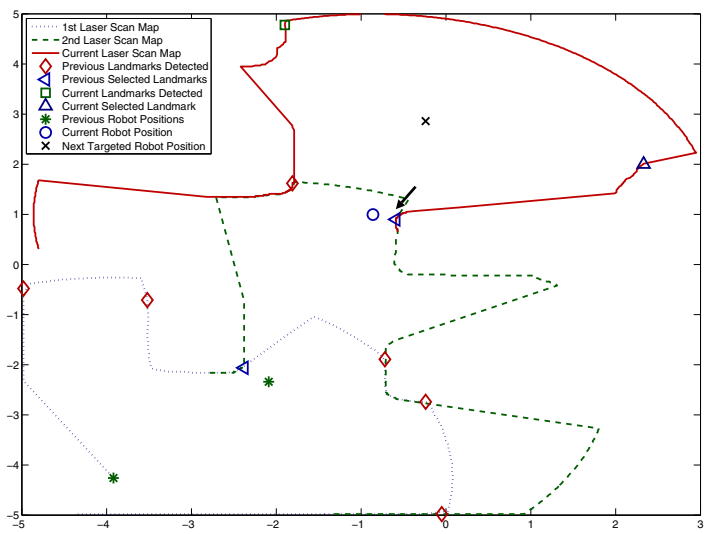

Figure 10. Third scan of the environment.

the final target.

Fig. 13 shows all previous laser scans and the route of the robot as calculated by its localisation system and as it appears by the GPS system. From the graph it is seen that the autonomous agent has performed quite well in the first scans. Nevertheless, there is a small drifting in the accuracy of the localisation system in the last phase of the navigation process caused mainly by the noise. In this last phase the landmarks selected, as can be seen from Fig. 13, are not prominent corners, but they rather occur in a curvy slope.

\section{Conclusions and future work}

In this paper the problem of localisation and mapping has been addressed. The key idea behind our work is that no

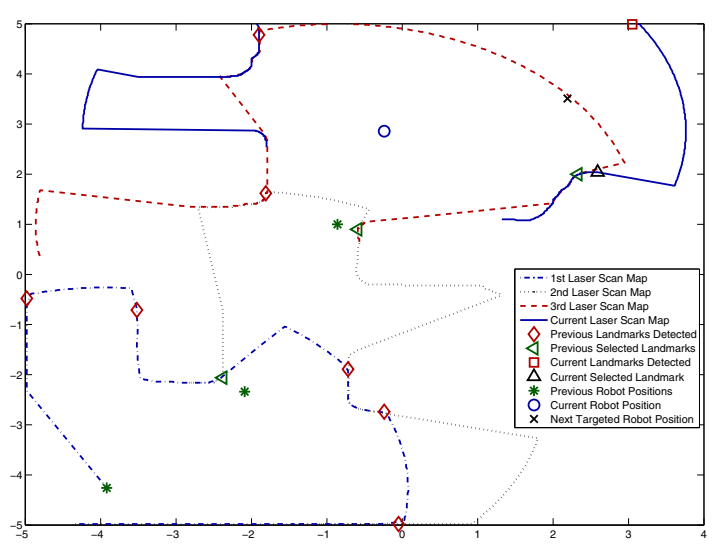

Figure 11. Fourth scan of the environment.

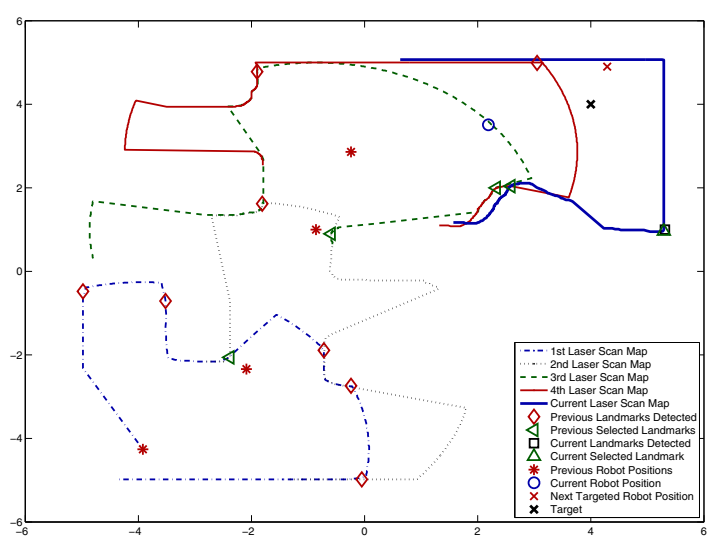

Figure 12. Fifth scan of the environment.

prior knowledge of the environment is used nor any odometry information. In fact, the odometry has been substituted by a laser range scanner. Its main purpose is to localise a robotic agent and map the environment while at the same time selecting efficient paths for driving the robotic agent to a target place. An inherent behaviour is obstacle avoidance. This is performed while path planning obstacle-free routes to desired locations.

The problem of localisation and mapping is at the heart of robot navigation and it has been approached through many ways. Our method promises to be efficient and accurate so long as the environment permits it. For example, an environment in which wide corridors occur results in erroneous estimates of the location of the robot. However, our approach is described by its simplicity and its effi- 


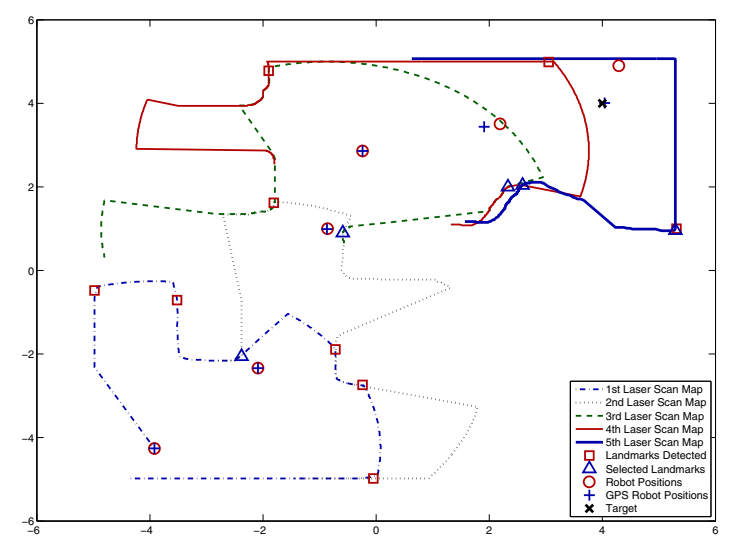

\section{Figure 13. A performance comparison be- tween the localisation system of the robot and the GPS.}

ciency. The simulated environment we have used to support our methodologies proves our assertions.

Future work will focus on larger scale and different types of environments. Moreover, we want to extract more salient features that will serve as landmarks. Last but not least, our work is part of a larger project where the navigation strategies are adapted according to the information available. This piece of work is trying to tackle the localisation and map building problem with minimal sensing.

\section{References}

[1] F. Amigoni, S. Gasparini, and M. Gini. Map building without odometry information. In Proceedings of the IEEE International Conference on Robotics and Automation, volume 4, pages 3753-3758, 2004.

[2] D. An and H. Wang. VPH: A new laser radar based obstacle avoidance method for intelligent mobile robots. In Proceedings of the Fifth World Congress on Intelligent Control and Automation (WCICA), volume 5, pages 4681-4685, 2004.

[3] L. Armesto and J. Tornero. SLAM based on Kalman filter for multi-rate fusion of laser and encoder measurements. In Proceedings of the IEEE/RSJ International Conference on Intelligent Robots and Systems, volume 2, pages 1860-1865, 2004.

[4] M. Choi, R. Sakthivel, and W. K. Chung. Neural networkaided extended Kalman filter for SLAM problem. In Proceedings of the IEEE International Conference on Robotics and Automation, pages 1686-1690, 2007.

[5] A. Davison, I. Reid, N. Molton, and O. Stasse. MonoSLAM: Real-time single camera SLAM. IEEE Transactions on Pattern Analysis and Machine Intelligence, 29(6):1052-1067, 2007.
[6] G. Dissanayake, P. Newman, S. Clark, H. F. Durrant-Whyte, and $\mathrm{M}$. Csorba. A solution to the simultaneous localization and map building (SLAM) problem. IEEE Transactions on Robotics and Automation, 17(3):229-241, 2001.

[7] T. Einsele. Real-time self-localization in unknown indoor environments using a panorama laser range finder. In Proceedings of the IEEE/RSJ International Conference on Intelligent Robots and Systems, pages 697-702, 1997.

[8] D. Fox, S. Thrun, W. Burgard, and F. Dellaert. Particle filters for mobile robot navigation. In A. Doucet, N. de Freitas, and N. Gordon, editors, Sequential Monte Carlo methods in practice. Springer, 2001.

[9] B. P. Gerkey, R. T. Vaughan, and A. Howard. The Player/Stage project: Tools for multi-robot and distributed sensor systems. In Proceedings of the 11th International Conference on Advanced Robotics, pages 317-323, Coimbra, Portugal, 2003.

[10] D. Hahnel, W. Burgard, D. Fox, K. Fishkin, and M. Philipose. Mapping and localization with RFID technology. In Proceedings of the IEEE International Conference on Robotics and Automation, volume 1, pages 1015-1020, 2004.

[11] D. Hahnel, W. Burgard, D. Fox, and S. Thrun. An efficient FastSLAM algorithm for generating maps of largescale cyclic environments from raw laser measurements. In Proceedings of the IEEE/RSJ International Conference on Intelligent Robots and Systems, volume 1, pages 206-211, 2003.

[12] Y. Koren and J. Borenstein. Potential field methods and their inherent limitations for mobile robot navigation. In Proceedings of the IEEE Conference on Robotics and Automation, pages 1398-1404, 1991.

[13] J. Leonard, H. F. Durrant-Whyte, and I. Cox. Dynamic map building for an autonomous mobile robot. The International Journal of Robotics Research, 11(4):286-298, 1992.

[14] J. L. Martinez, A. Pozo-Ruz, S. Pedraza, and R. Fernandez. Object following and obstacle avoidance using a laser scanner in the outdoor mobile robot Auriga- $\alpha$. In Proceedings of the IEEE/RSJ International Conference on Intelligent Robots and Systems, volume 1, pages 204-209, October 13-17 1998.

[15] M. Montemerlo, S. Thrun, D. Koller, and B. Wegbreit. FastSLAM: A factored solution to the simultaneous localization and mapping problem. In Proceedings of the AAAI National Conference on Artificial Intelligence, 2002.

[16] R. C. Smith and P. Cheeseman. On the representation and estimation of spatial uncertainty. International Journal of Robotics Research, 5(4):56-68, 1986.

[17] S. Thrun, D. Fox, and W. Burgard. Monte carlo localization with mixture proposal distribution. In Proceedings of the AAAI National Conference on Artificial Intelligence, 2000.

[18] S. Thrun, D. Fox, W. Burgard, and F. Dellaert. Robust Monte Carlo localization for mobile robots. Journal of Artificial Intelligence, 128(1-2):99-141, 2001.

[19] I. Ulrich and J. Borenstein. VFH+: Reliable obstacle avoidance for fast mobile robots. In Proceedings of the 1998 IEEE International Conference on Robotics and Automation, pages 1572-1577, Leuven, Belgium, May 16-21 1998. 